\title{
Disability Evolution in Multiple Sclerosis: How to Deal with Missing Transition Times in the Markov Model?
}

\author{
V. Petiot ${ }^{a} \quad$ C. Quantin ${ }^{a} \quad$ G. Le Teuff ${ }^{a} \quad$ M. Chavance ${ }^{c} \quad$ C. Binquet $^{a}$ \\ M. Abrahamowicz ${ }^{\text {d, e }}$ T. Moreau ${ }^{\text {b }}$

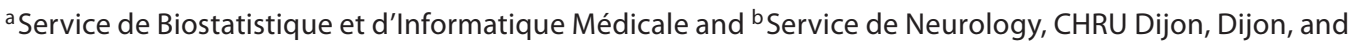 \\ 'Inserm, U472, Paris, France; ${ }^{\mathrm{d}}$ Department of Epidemiology and Biostatistics, McGill University and \\ e Division of Clinical Epidemiology, McGill University Health Centre, Montreal, Canada
}

\section{Key Words}

Multiple sclerosis · Markov model $\cdot$ Missing data $\cdot$ Multiple imputation

\begin{abstract}
Markov modeling of disability progression in multiple sclerosis requires knowledge of all times of transitions from a given level of disability to the next level, but such data are often missing. We address methodological challenges due to partly missing transition times. To estimate the effects of prognostic factors on the risk of transitions between three consecutive disability levels, two methods were used to deal with missing data. Listwise deletion limited the analysis to subjects with complete data. Multiple imputation of missing data revealed that data were missing at random (MAR mechanism) and imputed the missing transition times from the Weibull model. The results were then compared with the full data set with the actual times established through chart review. Multiple imputation estimates were systematically closer to those from the full data set than the listwise deletion estimates.

Copyright $\odot 2007$ S. Karger AG, Basel
\end{abstract}

\section{KARGER}

Fax +41613061234 E-Mail karger@karger.ch www.karger.com
(C) 2007 S. Karger AG, Base

0251-5350/07/0281-0056\$23.50/0

Accessible online at:

www.karger.com/ned

\section{Introduction}

Multiple sclerosis (MS) is the most frequent chronic disabling disease of the central nervous system in adults in western countries. This disease mainly affects young people and particularly women aged from 20 to 35 years. MS induces a full spectrum of disease ranging from benign, and even asymptomatic, to more severe cases including wheelchair dependence [1]. Consequently, the MS course is usually assessed through the progression of disability measured by means of disability scales such as the Expanded Disability Status Scale (EDSS) [2] or the European Database for Multiple Sclerosis (EDMUS) Grading Scale (EGS), which are concordant [3].

MS is characterized by two types of clinical events: relapse and progression. Relapses are defined as the appearance, the reappearance, or the worsening of symptoms of neurological dysfunction lasting more than $24 \mathrm{~h}$. Between these relapses, the neurological status of the patient can return to normal or present sequelae [1]. Progression is characterized by a continuous neurological deterioration for a minimum of 6 months [4]. For $85 \%$ of patients, at its onset MS is diagnosed as relapsing-remit-
Catherine Quantin, $\mathrm{MD}, \mathrm{PhD}$

Service de Biostatistique et Informatique Médicale, CHRU Dijon

1, boulevard Jeanne d'Arc, BP 77908

FR-21079 Dijon Cedex (France)

Tel. +33 3802936 29, Fax +33 3802939 73, E-Mail catherine.quantin@chu-dijon.fr 
ting MS. In half of the patients, relapsing-remitting MS evolves after about 10 years to a secondary progressive form of MS [4]. The remaining $15 \%$ of patients, mainly aged over 40 years, present a primary progressive MS, which is defined as a rapid disease progression from onset to disability [5].

A typical evolution of disability is rather well described: it takes an average of 8 years for patients to have a limited walking distance, 20 years to walk with unilateral support, and 30 years to become wheelchair dependent [6]. However, for individual patients the MS course remains at the present time unpredictable. Some studies have identified prognostic factors for MS progression. An older age at MS onset ( $>40$ years old), not presenting optic neuritis as the initial symptom of MS (compared to neurological long tract impairment) and a primary progressive MS at onset, are all associated with a worse evolution [6-16]. The degree of recovery after the first relapse, the time to a second relapse, and the number of exacerbations during the first 5 years after MS onset also predict the disability evolution [1-6, 17]. These characteristics seem to be strong predictors until the onset of walk impairment (EDSS score equal to 4). In contrast, they lose their predictive ability once score 4 has been reached [9].

In most of these studies, the statistical analysis uses multivariable time-to-event methods. Wolfson and Confavreux $[18,19]$ and Confavreux et al. [20] proposed to use a new approach, based on Markov multistate modeling, to study the clinical course of MS, which is described by 'the movement of patients through well-defined disease states'. These states were defined according to the different forms of the disease: pure relapse (without sequelae), relapse with sequelae, progression, death and loss to follow-up. We propose here to use a similar approach, based on a Markov model [21], but where the states are defined by the consecutive EGS scores, to identify the prognostic factors for disability progression. However, this approach requires information on the time of transition from one state to another and, sometimes, this information may be missing due to measurement problems. A systematic review of the medical files is one solution but in large databases the time-consuming work necessary to recover the original information could be impossible. A possible alternative is to replace the missing values by appropriate values, computed by multiple imputations $[22$, 23 . To address this problem of missing data on some transition times, the objective of this paper is to assess the potential advantages of multiple imputations in the context of Markov modeling of MS progression.

\section{Materials and Methods}

\section{Data}

The study population consisted of 313 patients with clinically or laboratory-supported MS defined according to the Poser criteria [24], and followed in the Neurology Department of the teaching hospital of Dijon from 1990 to 2003 . They were included in the EDMUS [1] used in all European countries. Clinical and therapeutic data were collected during each visit or hospitalization. Patients had at least one annual consultation. If the patient was first seen in the department after the onset of the disease, data were entered retrospectively after an interview with structured questions, designed according to standardized criteria to identify each step of the disease progression and validated by neurologists or general practitioners. The information in the database was derived by neurologists directly from medical files, and included automatic controls to reduce the frequency of incomplete or incorrect data. All the recorded information was also verified by another neurologist against the medical file. Data were updated weekly and they were automatically checked, for their consistency with previous information. An effort was consistently made to obtain data from the original clinical files. This was facilitated by the regular contacts with the MS regional network of Burgundy which covers the entire area. Confidentiality and safety of the data were ensured in agreement with the recommendations of the French Commission Nationale Informatique et Libertés (CNIL), which also provided approval. All patients gave informed consent for the inclusion of their data in the database.

Individual case reports included identification and demographic data, medical history, key episodes and dates in the MS course (relapses, onset of the progressive course, times when the patient reached the successive states of irreversible disability), biological and electrophysiological examination, magnetic resonance imaging data, concomitant diseases including (1) inflammatory and immunological disease, allergy, (2) cardiovascular disease, (3) toxic disease, (4) other neurological diseases (epilepsy, migraine) and psychological or psychiatric disorders, (5) endocrine and metabolic disease, (6) cancer and (7) trauma, as well as treatments and adverse events. In the study, we evaluated the prognostic value of the following covariates on the disability course: gender, age at MS onset (years), symptoms at MS onset (optic neuritis, long tract symptoms or brainstem symptoms), and disease type at onset (progressive or remitting).

The course of disability was represented by transitions through states of increasing disability, defined by EGS scores. The disability was measured according to the EGS [1], an ambulatory scale derived from the McAlpine classification [25] and from the EDSS scale [2]. EDMUS scores range from 0 (no neurological abnormality) to 10 (death from MS), with nine intermediary steps (see Appendix 1). A disability level was defined as irreversible when the criteria for a given EGS score had been reached and had persisted for at least 6 months, excluding any transient worsening of disability related to relapses.

Markov Multistate Modeling of MS Disability Progression

The course of disability was described by a Markov model. The Markov model extends the conventional time-to-event analysis to describe transitions between several consecutive or alternative states. In our study, the following three consecutive states were defined for the analysis: state 1: no or light disability (EGS scores 
Fig. 1. Multistate Markov model for MS disability progression.

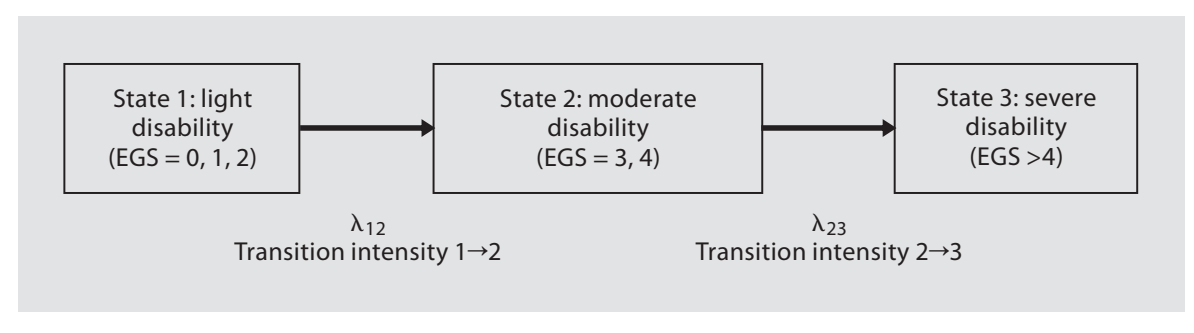

0,1 and 2); state 2: a moderate disability corresponding to a limited ability to run or walk more than $500 \mathrm{~m}$ (EGS scores 3 and 4); state 3: a severe disability (EGS scores $\geq 5$ ). At the onset of the study, patients were classified into state 1 , corresponding to light disability. Throughout the course of the disease, the patients can move from state 1 to state 2 and from state 2 to state 3 . Because of the irreversibility of the EGS score, transitions from a more severe disability level to a less severe state were not allowed in the analysis. Since a direct transition from state 1 to state 3 is theoretically possible, but very rare in practice, this transition was not considered in the analysis.

To model the intensities, i.e. risks of these transitions, we relied on a three-state time-homogeneous Markov model [26]. The transition intensity $\lambda_{\mathrm{jk}}$ can be interpreted as an 'instantaneous risk' of making a transition from state $j$ to state $k$ [27]. The homogeneity assumption implies that the instantaneous risk of transition between the two states is the same at any time during the follow-up [26]. To assess the impact of covariates on the probabilities of consecutive transitions, the time-homogeneous Markov model can be extended to the multivariable proportional intensity regression model [28]. In this model, a covariate $\left(\mathrm{Z}_{\mathrm{i}}\right)$ is assumed to affect the intensity of transition from state $j$ to state $k$ by a proportional factor $\left(\exp \left[\beta_{\mathrm{ijk}}\right]\right)$, so that a model with two transitions requires estimating two regression coefficients for each covariate. The effects of the different covariates are assumed to be multiplicative and constant over time, both assumptions being consistent with the conventional proportional hazards model [29]. Therefore, the interpretation of $\left(\exp \left[\beta_{\mathrm{ijk}}\right]\right)$ is similar to that of the hazard ratio. All baseline intensities and all regression coefficients were simultaneously estimated using the maximum likelihood criterion [28]. Figure 1 shows the three consecutive states considered in our Markov model [21] analyses and identifies the two transition intensities, used as the outcome variables in the multistate analysis.

\section{Missing Transition Times}

For some patients, we had recorded the times of both transitions ( 1 to 2 and 2 to 3 ) or of the first transition (1 to 2 ) only, and for all these patients the data were considered complete. However, for other patients we had only information on the time the patient had reached state 3 but not state 2 . For those subjects, we considered the time of reaching state 2 as missing. Notice that for these patients, times of both transitions 1 to 2 and 2 to 3 are missing, but both times would be known if we knew when they reached state 2 (fig. 1). Indeed, based on the arguments presented above, we assumed that a patient could not reach directly the most severe state (state 3 ) without having experienced before a moderate disability (state 2). On the other hand, if a patient was still in state 1 at the end of follow-up, we assumed this information was correct.
In our study, it was unlikely that we should miss the transition from state 1 to 2 because all the patients were regularly seen for consultation at least once a year.

\section{Identification of the Mechanism of Missing Data}

First, we attempted to characterize the missing data mechanism. This was performed by considering the observation of transition to state 2 as a binary variable equal to 1 if the time to reach state 2 was missing, and otherwise equal to 0 . Then, multiple logistic regressions were employed in order to check whether the occurrence of missing values was associated with some independent variables [30]. When no association exists, missing data are said to be missing completely at random (MCAR). Under the MCAR assumption, the missing data values are a simple random sample of all data values [23]. If some associations do exist but the probability of being missing depends only on the values of observed covariates, the missing data are considered missing at random (MAR). Finally, the mechanism is said to be missing not at random (MNAR) when the probability of being missing directly depends on the missing values. In our context, the MNAR assumption would hold if the missing transition times were systematically shorter or systematically longer than the observed (nonmissing) times. Like in most clinical studies, we did not take into account the MNAR process in the statistical analysis as it requires assumptions that generally cannot be checked [22].

\section{Alternative Approaches to Deal with Missing Transition \\ Times}

In further analysis, we considered two different approaches to deal with the missing data problem in the context of multivariable Markov modeling. Below, we briefly describe these two alternative approaches: (1) complete-cases-only analysis, corresponding to the listwise deletion of observations and (2) multiple imputations. Finally, we describe the procedure used to reconstruct the missing data, through re-examination of the medical files, which provided us with the reference against which the results of the two approaches were compared.

\section{Complete-Cases-Only Analysis (Listwise Deletion)}

Listwise deletion is the simplest approach used to deal with missing observations. It consists of ignoring all incomplete observations in the statistical analysis. This method, also referred to as the complete-cases-only analysis, is used by default in most statistical software packages.

Multiple Imputation of Missing Transition Times

The second method considered to deal with missing data involved multiple imputations [31]. It requires first using original 
incomplete data in order to build a prediction or imputation model, to estimate the missing values, here the time the patient reaches state 2. Following Rubin [22], all the available explanatory variables were used, regardless of their significance level, in order to limit the risk of MNAR data. Several statistical distributions were considered for the transition time from state 1 to state 2 (exponential, log-normal, Weibull, standard gamma, generalized gamma). These distributions are all nested within the generalized gamma model, making it a simple matter to evaluate them with the likelihood ratio test and, thus, allowing us to select the simplest distribution consistent with the observed data.

At the second step of the analyses, the selected imputation model is used to replace the missing values by plausible ones, taking into account the different levels of uncertainty about the true values. In order to take into account the uncertainty about the model coefficients, $\mathrm{M}$-completed data sets are built, each with a different set of parameters drawn at random, according to the asymptotic distribution of the corresponding estimator. Since the prediction model is not deterministic but stochastic, the imputed values are drawn from the statistical distribution provided by these model coefficients and by the explanatory variables. Five imputations $(M=5)$ were performed, as suggested by Allison [32], resulting in five completed data sets. The five imputed data sets were then analyzed separately, using the Markov model described in the previous section, and their results were combined according to Rubins's formula [23].

The combined variance $\left(S d_{c}^{2}\right)$ of the multiple imputation estimator is the sum of an interimputation and of an intraimputation component estimated as:

$$
S d_{c}^{2}=\frac{1}{M} \sum_{k=1}^{M} S_{k}^{2}+\left(1+\frac{1}{M}\right)\left(\frac{1}{M-1}\right) \sum_{k=1}^{M}\left(\beta_{k}-\beta_{0}\right)^{2}
$$

where $S_{k}^{2}$ is the asymptotic intraimputation variance of the estimator provided by imputation $\mathrm{k}$.

\section{Comparing the Performance of Multiple Imputation and Complete Case Analyses}

Next, we attempted to assess the potential advantages of multiple imputation against the conventional analyses, restricted to complete cases only. To this end, we performed an in-depth review of medical records for those patients for whom the time of reaching state 2 was not observed. If no sufficient information was obtained, the patients themselves or their neurologists were contacted. The patient was considered as having evolved toward state 2 at time $t$, if this disability level was observed at time $t$, as well as at a further visit, at least 6 months after time t. This allowed us to create the 'full data set', in which all transition times of interest were considered known.

Markov modeling of the two transitions was then repeated separately in three different data sets: (1) the 'full data set', (2) the 'complete-cases-only subset' that included only those patients who did not have missing transition times in the original data, and (3) the 'imputed data set', which included all study subjects and where the originally missing transition times were replaced by the values obtained through multiple imputation. The results of the full data set analyses were considered the reference, against which the estimates obtained in the two other analyses were compared. In particular, in the complete-cases-only and multiple imputation analyses, we calculated the difference in the regression coeffi-
Table 1. Patient characteristics at the first relapse

\begin{tabular}{lc}
\hline Characteristics & \\
\hline Patient distribution & \\
$\quad<40$ years & $232(74.1)$ \\
$\geq 40$ years & $81(25.9)$ \\
\hline Patient distribution & \\
$\quad<20$ years & $31(9.9)$ \\
$20-40$ years & $201(64.2)$ \\
$\quad \geq 40$ years & $81(25.9)$ \\
\hline Age, years & $32.7 \pm 10.9$ \\
Gender & $85(27.2)$ \\
Men & $228(72.8)$ \\
Women & \\
\hline Symptomatology & \\
Monosymptomatic & $253(80.8)$ \\
Polysymptomatic & $60(19.2)$ \\
\hline Long tract symptoms & \\
Yes & $185(59.1)$ \\
No & $128(40.9)$ \\
\hline Brainstem symptoms & \\
Yes & \\
No & \\
\hline Optic neuritis & \\
Yes & \\
No & $214(31.6)$ \\
\hline Disease type & \\
Remittent & \\
Progressive & \\
\hline Concomitant pathologyb & \\
$\quad$ Nes & \\
No & \\
\hline
\end{tabular}

With the exception of age (mean \pm SD) the values represent number with the percentage in parentheses.

a Patients are classified as 'polysymptomatic' if they have at least 2 different symptoms.

${ }^{\mathrm{b}}$ Concomitant pathology includes (1) inflammatory and immunological disease, allergy, (2) cardiovascular disease, (3) toxic disease, (4) other neurological diseases (epilepsy, migraine) and psychological or psychiatric disorders, (5) endocrine and metabolic disease, (6) cancer and (7) trauma. 
Fig. 2. Number of transitions recorded in the original, incomplete database $(n=313$ patients). $\mathrm{n}_{\mathrm{ij}}$ represents number of patients from state i to state $\mathrm{j}(\mathrm{i}=1,2 ; \mathrm{j}=1, \ldots, 3$ with $\mathrm{i} \leq \mathrm{j}$; dashed line corresponds to the observed transition from state 1 directly to state 3 with missing information on the time of reaching state 2 ; solid lines correspond to the observed transitions, circles indicating patients who remained in a given state until the end of their follow-up.

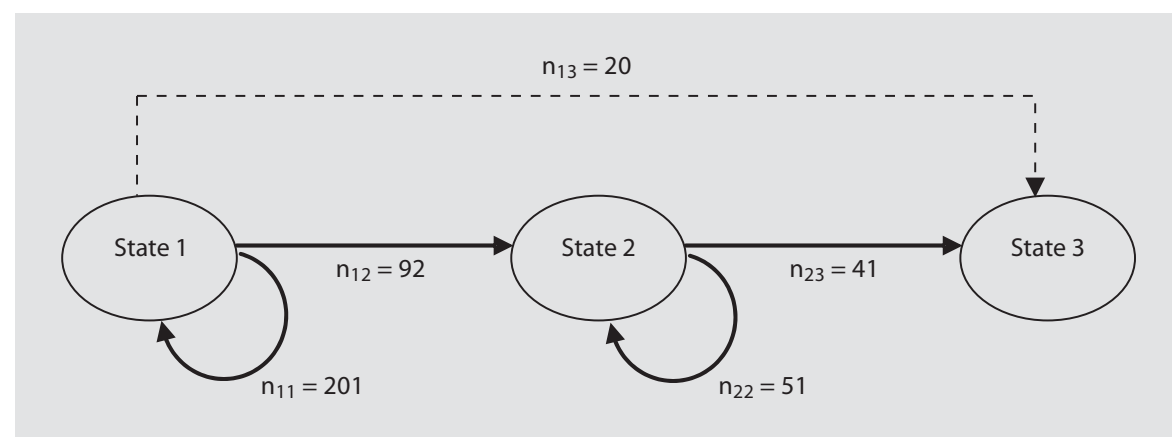

Table 2. Identification of the missing data mechanism

\begin{tabular}{|c|c|c|}
\hline Covariates & Odds ratio ${ }^{a}(95 \% \mathrm{CI})$ & $\mathrm{p}$ value \\
\hline \multicolumn{3}{|l|}{ Patient distribution } \\
\hline$<20$ years & 1 & \\
\hline 20-40 years & $0.742(0.332-1.656)$ & 0.4663 \\
\hline$\geq 40$ years & $0.777(0.297-2.037)$ & 0.6085 \\
\hline \multicolumn{3}{|l|}{ Gender } \\
\hline Men & 1 & \\
\hline Women & $0.735(0.424-1.275)$ & 0.2735 \\
\hline \multicolumn{3}{|l|}{ Symptomatology } \\
\hline Monosymptomatic & 1 & \\
\hline Polysymptomatic & $1.167(0.691-1.972)$ & 0.5633 \\
\hline \multicolumn{3}{|l|}{ Disease type } \\
\hline Remittent & 1 & \\
\hline Chronic & $2.314(1.035-5.173)$ & 0.0410 \\
\hline \multicolumn{3}{|l|}{ Concomitant pathology ${ }^{c}$} \\
\hline Yes & 1 & \\
\hline No & $0.314(0.106-0.930)$ & 0.0366 \\
\hline Unknown & $0.394(0.129-1.209)$ & 0.1034 \\
\hline $\begin{array}{l}{ }^{\mathrm{a}} \text { Odds ratio }>1 \text { indi } \\
\text { data. } \\
\text { b The different sym } \\
\text { symptoms, optic neurit } \\
\text { ate (mono-, polysymptc } \\
{ }^{c} \text { Concomitant path } \\
\text { munological disease, al } \\
\text { disease, (4) other neur } \\
\text { psychological or psych } \\
\text { bolic disease, (6) cance }\end{array}$ & $\begin{array}{l}\text { higher probability of ha } \\
\text { s (long tract symptom } \\
\text { ve been aggregated int } \\
\text { c). } \\
\text { includes (1) inflamma } \\
\text { (2) cardiovascular dise } \\
\text { al diseases (epilepsy, m } \\
\text { disorders, (5) endocrir } \\
\text { (7) trauma. }\end{array}$ & $\begin{array}{l}\text { missing } \\
\text { ainstem } \\
\text { e covari- } \\
\text { and im- } \\
\text { (3) toxic } \\
\text { ine) and } \\
\text { d meta- }\end{array}$ \\
\hline
\end{tabular}

cients, for each covariate and each of the two transitions, between the estimated coefficient and the corresponding estimate from the full data set. To assess the importance of these differences, we divided them by the standard error (SE) from the full data set analysis. A difference bigger than 1 SE was considered 'important'. In addition, we calculated the ratios of the SE for each coefficient ob- tained from the two methods for handling missing data to the corresponding SE from the full data set analyses, to assess the possible loss of precision induced by the missing transition times.

MKVPCI v1.0 program [21] was used to estimate the Markov model parameters. Parametric regression model for the multiple imputations was implemented using the Lifereg procedure of SAS software v8.2.

\section{Results}

The characteristics of the study population are presented in table 1 . Among the 313 patients included, 73\% were women. Mean age at MS onset was 33 years (SD = 11 years); $26 \%$ of the patients were over 40 . The symptoms at the first relapse involved long tracts in $59 \%$ of the cases, and brainstem in $32 \%$ of the cases; $28 \%$ of the patients presented optic neuritis. Overall, $81 \%$ of the study patients were monosymptomatic. The MS onset had a relapsing-remitting form in $92 \%$ of the subjects.

Figure 2 shows the transitions recorded in the original, incomplete database. Among 61 patients, for whom the time of reaching state 3 was recorded, 20 (32.7\%) had no information on the time when they reached state 2 .

Table 2 presents the results of multiple logistic analyses aimed at identifying the missing data mechanism. The odds ratios shown in table 2 assess the association between a given covariate and the odds of the patient having missing information at the time of reaching state 2. Chronic disease, as opposed to its remittent form, and the presence of a concomitant pathology are both associated with the significantly higher probability of missing data (table 2 ). Thus, the missing data mechanism corresponds to the MAR process. As a consequence, a parametric regression model was built to link the expected time of transition from state 1 to state 2 , i.e. of reaching state 2 , with relevant patient's characteristics. Among the different parametric distributional models considered, the Weibull distribution 
Table 3. Comparison of the estimated parameters (standard errors) in Markov model using listwise and multiple imputation

\begin{tabular}{|c|c|c|c|c|c|c|}
\hline \multirow[t]{2}{*}{ Covariates } & \multicolumn{2}{|l|}{ Full data set ${ }^{\mathrm{a}}$} & \multicolumn{2}{|c|}{ Listwise deletion $^{\mathrm{b}}$} & \multicolumn{2}{|c|}{ Multiple imputation ${ }^{c}$} \\
\hline & $1 \rightarrow 2(112)^{d}$ & $2 \rightarrow 3(61)^{\mathrm{e}}$ & $1 \rightarrow 2(92)^{d}$ & $2 \rightarrow 3(41)^{\mathrm{e}}$ & $1 \rightarrow 2(112)^{d}$ & $2 \rightarrow 3(61)^{\mathrm{e}}$ \\
\hline Women vs. men & $-0.282(0.213)$ & $0.249(0.309)$ & $-0.229(0.213)$ & $-0.003(0.307)$ & $-0.313(0.229)$ & $0.184(0.353)$ \\
\hline Age (years) & $-0.002(0.009)$ & $0.009(0.014)$ & $-0.005(0.009)$ & $0.034(0.014)$ & $-0.001(0.009)$ & $0.004(0.013)$ \\
\hline Long tract vs. optic neuritis & $0.118(0.255)$ & $-0.121(0.374)$ & $0.069(0.256)$ & $0.272(0.364)$ & $0.026(0.260)$ & $0.113(0.391)$ \\
\hline Brainstem vs. optic neuritis & $0.541(0.279)$ & $-0.479(0.372)$ & $0.435(0.279)$ & $-0.083(0.383)$ & $0.534(0.281)$ & $-0.217(0.385)$ \\
\hline Chronic vs. remittent & $0.391(0.314)$ & $-0.425(0.425)$ & $0.379(0.317)$ & $-0.812(0.436)$ & $0.423(0.328)$ & $-0.277(0.438)$ \\
\hline
\end{tabular}

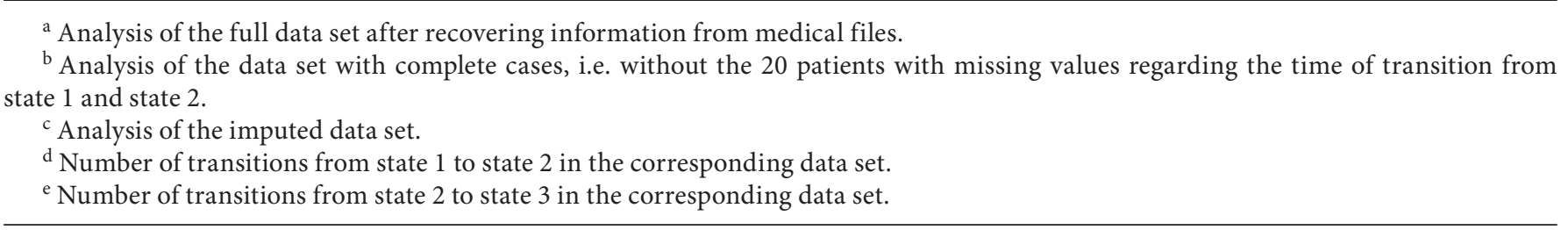

was most consistent with the observed distribution of the dependent variable, i.e. the time of transition from state 1 to state 2 , and was selected for multiple imputations.

Table 3 compares the results of Markov modeling of the two transitions ( 1 to 2 and 2 to 3 ) obtained with three different sets of data. The results for (1) the full data set (with missing transition times reconstructed from a detailed medical chart review) are considered the reference, against which the estimates obtained from (2) the complete-cases-only subset (with listwise deletion of subjects with missing data), and (3) the multiple imputation of the missing transition times are compared. In general, the results from the three analyses are quite concordant in the case of transition 1 to 2 (table 3 ). In contrast, for transition 2 to 3 the complete-cases-only (listwise deletion) estimates diverge often from the results of the full data set analyses. For example, the first row of table 3 suggests that women have higher intensity of transition 2 to 3 than men (regression coefficient of 0.249 for full data set). In contrast, the effect of gender on the intensity of transition 2 to 3 is practically null $(-0.003)$ in the complete-casesonly analysis, while the estimate obtained from multiple imputation (0.184) is much closer to the 'reference value' from the full data set. On the other hand, the estimated 'protective' effect of having the chronic rather than the remittent form of the disease on transition 2 to 3 seems much stronger in complete-cases-only analyses $(-0.812)$ than in the full data set $(-0.425)$. Here, again, the estimate obtained from multiple imputation $(-0.277)$ is substantially closer to the estimate from the full data set than that yielded by listwise deletion. Finally, the complete-casesonly analysis yielded a statistically significant impact of older age on the risk of transition 2-3 ( $\mathrm{p}=0.015)$, in contrast to a practically null effect obtained with both full data set and multiple imputation (2nd row of table 3).

Overall, in almost all cases, the multiple imputation results agree well with the estimates from the full data set, with the difference between the two estimates always much lower than 1 SE of the reference (full data set estimate). The only case where the point estimate for multiple imputation has a different sign from the 'reference value' concerns the association of long tracts with transition 2 to 3. However, this apparent difference in the point estimates is likely to be explained by sampling errors as it corresponds to less than 0.6 of the reference SE $(0.234 / 0.374)$, i.e. can be considered only 'moderate' according to statistical criteria. Most importantly, the difference between the negative coefficient from the full data set $(-0.121)$ and the positive estimate from multiple imputation $(+0.113)$ becomes practically irrelevant once we realize that both estimates are statistically definitely nonsignificantly different from 0 . Finally, even in this case the multiple imputation (MI) estimate of +0.113 is considerably less divergent from the reference value of -0.121 than the estimate obtained from listwise deletion (+0.272), even if the latter is also statistically nonsignificant.

In general, the complete-cases-only analysis of the subset obtained through listwise deletion systematically yielded results more discrepant from the full data set analyses than the corresponding MI-based estimates (table 3). In many cases, the listwise deletion estimates for transition 2 to 3 differed from the reference values by more than $1 \mathrm{SE}$ of the latter (e.g. for age, long tracts, brainstem), indicating 'important differences'. 
Finally, table 3 also shows that the SE obtained from the three analyses were very similar. Still, MI yielded marginally higher SEs than the full data set, which reflected additional uncertainty due to imputation.

\section{Discussion}

Prospective clinical studies of the evolution of MS disability are important to further explore the role of putative prognostic factors [33-39]. In this context, Markov multistate modeling has the advantage of being able to represent the entire longitudinal trajectory of a patient's progressively increasing disability, and to account for a possibly different impact of a given factor on the risks of transitions between different levels of disability $[18,19]$. On the other hand, Markov modeling of MS disability progression requires complete data on individual times when particular patients have reached consecutive disability levels. Yet, in clinical practice, these times may be unknown for some transitions of a subset of patients.

In this article, we present an empirical investigation of the methodological issues related to incomplete data on times of transitions between specific disability levels. In particular, we report on Markov modeling of the progression between three consecutive states, corresponding to increasing levels of MS disability, defined by the EDMUS scores [3]. The data set we analyze includes $313 \mathrm{MS}$ patients, among whom 20 (6.3\%) have missing data on the time of reaching the intermediate state 2 even if they have recorded the time when they were assigned the severe disability, corresponding to state 3 . Thus, for these patients neither the time for transition from state 1 to state 2, nor from state 2 to 3 are known. We apply two different statistical methods to deal with such incomplete observations for reaching state 2 . The first approach considered the involved conventional listwise deletion method, which reduced the data set for the analysis by simply excluding all the patients with any missing transition times. In contrast, the multiple imputation methodology [22, $23,31,32]$ permitted both exploring the mechanism generating the missing data, and imputing plausible values for missing transition times.

To assess the performance of the two methods, we first reconstructed the full data set. Specifically, we carried out an in-depth review of the medical files for patients with originally missing data and, on this basis, established when each patient reached state 2 . We then compared the results of the Markov multistate analyses of the full data set with those obtained in data sets created through (1) listwise deletion of patients with missing transition times, and (2) multiple imputations. We focused on the differences between the corresponding estimates of the effects of various prognostic factors on the intensity of each of the two transitions, and considered the estimates from the full data set as the reference.

Several estimates yielded by the analysis of the complete-cases-only subset, resulting from the listwise deletion, diverged importantly from the corresponding reference values. In particular, in the case of transition 2 to 3 , several differences between the listwise deletion and the full data set estimates were close to or even exceeded $1 \mathrm{SE}$ of the reference coefficient. This pattern of results was expected given that listwise deletion is likely to induce not only loss of information but also systematic selection bias [23]. Indeed, in our context such selection bias is corroborated by the fact that multiple logistic regression analyses revealed that the proportion of incomplete observations was significantly higher among patients with chronic disease and with concomitant pathology.

In contrast to the simple listwise deletion, multiple imputation required additional analyses to (1) explore the type of the mechanism that generates missing data, (2) find the appropriate distribution of the transition times, (3) build a parametric model to predict the expected values of the missing times, based on patients' observed characteristics, and (4) account for the uncertainty regarding the true values. In our study, these additional analyses paid off, as the estimates obtained through multiple imputations were systematically closer to the reference values than those obtained through listwise deletion. Even if for long tracts the sign of the point estimate was different in the full data set than for multiple imputations, this apparent discrepancy was practically irrelevant as both estimates were statistically completely nonsignificant. Moreover, in contrast to complete-cases-only results, the differences between the multiple imputation and the full data set regression coefficients never approached or exceed 1 SE.

One of the reasons why, in our study, multiple imputation performed well was that the first step of the analyses confirmed that the missing data mechanism corresponded to the MAR assumption. This allowed us to relate the expected values of the missing transition times to the observed patients' characteristics. If such relationships did not exist, the missing data would have to be considered as MCAR data, in which case a reasonable strategy would involve imputing simply the mean of the observed (nonmissing) times for all patients with missing data. Actually, the MCAR assumption corresponds to a special case 
of the MAR data, with the imputation model reduced to the intercept only, i.e. to the constant corresponding to the unconditional mean of all observed values. If, as it was the case in our analyses, the missing values do depend on some observed covariates, then predicting the values from the multivariable model, i.e. making the imputed value conditional on these covariates, clearly improves the performance of the multiple imputation method.

Another issue that should be addressed by future methodological and empirical studies involves the robustness of the results, related to the advantages of multiple imputation, with respect to the proportion of missing data. In our study, 20 of the total of 61 patients who reached state 3 had missing times of reaching state 2 . Thus, the proportion of missing times for transition 2 to 3 was $32.7 \%$ (20/61). The same 20 patients had missing times for transition 1 to 2 , but here the denominator was much higher, as a total of 112 patients (92 with known times 1 to 2 and 20 with missing data) reached state 2 . So the proportion of missing times for transition 1 to 2 was only $20 / 112$, i.e. $17.8 \%$. The fact that while the (absolute) number of missing times was the same for both transitions, their (relative) proportion was much higher for transition 2 to 3 may help interpreting the results shown in table 3. For transition 1 to 2 both listwise deletion and multiple imputation yielded the estimates quite close to the full data set analyses. In contrast, for transition 2 to 3 the estimates obtained through multiple imputations were much closer to the reference values, estimated in the full data set. Clearly a small proportion of incomplete observations limit the risk of bias and thus the potential benefits of multiple imputation. On the other hand, if the proportion of missing data is very high, then the number of subjects with nonmissing values of the outcome of interest may be insufficient to ensure accurate results of multiple imputations. In particular, with only a small number of observed (nonmissing) outcomes, it will be difficult to (1) establish which process generates the missing data, and (2) obtain stable estimates of the relationships between covariates and the observed values of the outcome variable. Simulation studies, in which the proportion of missing data and the underlying mechanism will be systematically varied, are necessary to gain further insights into these complex issues.

We also have to recognize some other limitations of our analyses. First, the limited number of outcomes, and especially of patients who reached state 3 (severe disability), have likely affected the statistical power of our Markov multistate analyses. This might explain why, even in the full data set, we failed to identify any prognostic fac- tors with statistically significant associations with the intensity of transition (table 3). For example, the lack of a significant impact of older age on the risk of transition from state 2 to 3 may be due to low statistical power, even if the association between age and MS progression was found in some studies [40] but not in others [41]. Second, we assumed that patients for whom there was no information on reaching either state 2 or state 3 remained truly in state 1 until the end of their follow-up time. Even if our close follow-up procedures made it unlikely that we missed the development of moderate disability, corresponding to state 2 , data could be missing on transition to state 2 for a few patients who were assumed to remain in state 1 . However, as discussed in the previous paragraph, a small proportion of missing data would have only a very minor impact on the results of both methods evaluated in our study.

\section{Conclusion}

Our results indicate the potential advantages of using multiple imputation to deal with missing transition times in Markov modeling of the MS disability evolution. Yet, simulation studies are necessary to provide a more systematic assessment of the performance of multiple imputations in this context, and to evaluate the robustness of its advantages across different proportions of missing values and different processes generating these values. Finally, confirmation of the substantive results of our analyses, regarding the impact of various prognostic factors, will require further empirical studies of different, especially larger, cohorts of MS patients.

\section{Appendix 1: EDMUS Grading Scale (EGS)}

0 Normal findings on neurological examination

1 No disability; minimal signs on neurological examination

2 Minimal and not ambulatory-related disability; able to run

3 Unlimited walking distance without rest, but unable to run, or a significant not ambulation-related disability

4 Walks without aid; limited walking distance, but $>500 \mathrm{~m}$ without rest

5 Walks without aid; limited walking distance, but $<500 \mathrm{~m}$ without rest

6 Walks with uni- or bilateral support; walking distance $<100 \mathrm{~m}$ without rest

7 Home-bound; a few steps with wall or furniture assistance; walking distance $<10 \mathrm{~m}$ without rest

8 Chair-bound; unable to take a step; some effective use of arms

9 Bedridden and totally helpless

10 Death due to MS 


\section{References}

1 Confavreux C, Aimard G, Devic M: Course and prognosis of multiple sclerosis assessed by the computerized data processing of 349 patients. Brain 1980;103:281-300.

2 Kurtze JF: Rating neurologic impairment in multiple sclerosis: an expanded disability status scale (EDSS). Neurology 1983;36:553564

-3 Confavreux C, Compston DA, Hommes OR, McDonald WI, Thompson AJ: EDMUS, a European database for multiple sclerosis. J Neurol Neurosurg Psychiatry 1992;55:671676.

4 Confavreux C: Relapses, progression, inflammation and neurodegeneration in multiple sclerosis: a changing view. Adv Clin Neurosci Rehabil 2002;2:7-9.

$\checkmark 5$ Bjartmar C, Wujek TR, Trapp BD: Axonal loss in the pathology of MS: consequences for understanding the progressive phase of the disease. J Neurosci 2003;206:165-171.

6 Confavreux C, Vukusic S, Moreau T, Adeleine P: Relapses and progression of disability in multiple sclerosis. N Engl J Med 2000; 343:1430-1438.

-7 Trojano M, Avolio C, Manzari C, Calo A, De Robertis F, Serio G, Livrea P: Multivariate analysis of predictive factors of multiple sclerosis course with a validated method to assess clinical events. J Neurol Neurosurg Psychiatry 1995;58:300-306.

$\checkmark 8$ Riise T, Gronning M, Fernandez O, Lauer K, Midgard R, Minderhoud JM, et al: Early prognostic factors for disability in multiple sclerosis, a European multicenter study. Acta Neurol Scand 1992;85:212-218.

-9 Runmarker B, Andersen O: Prognostic factors in a multiple sclerosis incidence cohort with twenty-five years of follow-up. Brain 1993;116:117-134.

>10 Weinshenker BG, Rice GP, Noseworthy JH, Carriere W, Baskerville J, Ebers GC: The natural history of multiple sclerosis: a geographically based study. 3. Multivariate analysis of predictive factors and models of outcome. Brain 1991;114:1045-1056.

-11 Weinshenker BG, Bass B, Rice GP, Noseworthy J, Carriere W, Baskerville J, et al: The natural history of multiple sclerosis: a geographically based study. 2. Predictive value of the early clinical course. Brain 1989;112: 1419-1428.

-12 Weinshenker BG, Bass B, Rice GP, Noseworthy J, Carriere W, Baskerville J, et al: The natural history of multiple sclerosis: a geographically based study. I. Clinical course and disability. Brain 1989;112:133-146.

13 Phadke JG: Clinical aspects of multiple sclerosis in north-east Scotland with particular reference to its course and prognosis. Brain 1990;113:1597-1628.
14 Phadke JG: Survival pattern and cause of death in patients with multiple sclerosis: results from an epidemiological survey in north east Scotland. J Neurol Neurosurg Psychiatry 1987;50:523-531.

15 Kurtzke JF, Beebe GW, Nagler B, Kurland LT, Auth TL: Studies on the natural history of multiple sclerosis. 8. Early prognostic features of the later course of the illness. Chronic Dis 1977;30:819-830.

16 McAlpine D: The benign form of multiple sclerosis. A study based on 241 cases seen within three years of onset and followed up until the tenth year or more of the disease. Brain 1961;84:186-203.

17 Cottrell DA, Kremenchutzky M, Rice GPA, Koopman WJ, Hader W, Baskerville A, et al: The natural history of multiple sclerosis: a geographically based study. 5. The clinical features and natural history of primary progressive multiple sclerosis. Brain 1999;122: 625-639.

18 Wolfson C, Confavreux C: A Markov model of the natural history of multiple sclerosis. Neuroepidemiology 1985;4:227-239.

19 Wolfson C, Confavreux C: Improvements to a simple Markov model of the natural history of multiple sclerosis. Neuroepidemiology 1987;6:101-115.

20 Confavreux C, Vukusic S, Adeleine P: Early clinical predictors and progression of irreversible disability in multiple sclerosis: an amnesic process. Brain 2003;126:770-778.

21 Alioum A, Commenges D: MKVPCI: a computer program for Markov models with piecewise constant transition intensities and covariates. Comput Methods Programs Biomed 2001;64:109-119.

22 Rubin DB: Multiple imputation after 18+ years. J Am Stat Assoc 1996;91:473-489.

23 Little RJA, Rubin DB: Statistical Analysis with Missing Data, ed 2. Hoboken, WileyInterscience, 2002.

24 Poser CM, Paty DW, Scheinberg L, McDonald WI, Davis FA, Ebers GC, et al. New diagnostic criteria for multiple sclerosis: guidelines for research protocols. Ann Neurol 1983;13:227-231.

25 McAlpine D: The benign form of multiple sclerosis. A study based on 241 cases seen within three years of onset and followed up until the tenth year or more of the disease. Brain 1961;84:186-203.

26 Andersen PK: Multistate models in survival analysis: a study of nephropathy and mortality in diabetes. Stat Med 1988;7:661-670.

27 Kalbfeish JD, Lawless JF: The analysis of panel data under a Markov assumption. J Am Stat Assoc 1985;80:863-871.
28 Marshall G, Guo W, Jones RH: Markov: a computer program for multi-state Markov models with covariates. Comput Methods Programs Biomed 1995;47:147-156.

29 Cox DR: Regression models and life tables (with discussion). J R Stat Soc B 1972;34:187220

30 Vercherin P, Gutknecht C, Guillemin F, Ecochard R, Mennen LI, Mercier M: Missing data mechanisms of the questionnaire SF36's items in the SU.VI.MAX study. Rev Epidemiol Sante Publique 2003;51:513-525.

-31 Rubin DB, Schenker N: Multiple imputation in health care databases: an overview and some applications. Stat Med 1991;10:585598.

32 Allison P: Missing Data. Iowa City, Sage University Papers Series, 2001.

33 Levic ZM, Dujmovic I, Pekmezovic T, Jarebinski M, Marinkovic J, Stojsavljevic N, Drulovic J: Prognostic factors for survival in multiple sclerosis. Mult Scler 1999;5:171178.

34 Pittock SJ, Mayr WT, McClelland RL, Jorgensen NW, Weigand SD, Noseworthy JH, Rodriguez M: Disability profile of MS did not change over 10 years in a populationbased prevalence cohort. Neurology 2004; 62:601-606.

35 Ghezzi A, Pozzilli C, Liguori M, Marrosu MG, Milani N, Milanese C, Simone I, Zaffaroni M: Prospective study of multiple sclerosis with early onset. Mult Scler 2002;8: 115-118.

-36 Eriksson M, Andersen O, Runmarker B: Long-term follow up of patients with clinically isolated syndromes, relapsing-remitting and secondary progressive multiple sclerosis. Mult Scler 2003;9:260-274.

37 Kantarci O, Siva A, Eraksoy M, Karabudak $\mathrm{R}$, et al: Survival and predictors of disability in Turkish MS patients. Turkish Multiple Sclerosis Study Group (TUMSSG). Neurology 1998;51:765-772.

38 Riise T, Gronning M, Aarli JA, Nyland H, Larsen JP, Edland A: Prognostic factors for life expectancy in multiple sclerosis analysed by Cox-models. J Clin Epidemiol 1988;41: 1031-1036.

39 Runmarker B, Andersson C, Odén A, Andersen $\mathrm{O}$ : Prediction of outcome in multiple sclerosis based on multivariate models. J Neurol 1994;241:597-604.

40 Confavreux C, Vukusic S: Age at disability milestones in multiple sclerosis. Brain 2006; 129:595-605.

41 Amato MP, Ponziani G, Bartolozzi ML, Siracusa G: A prospective study on the natural history of multiple sclerosis: clues to the conduct and interpretation of clinical trials. J Neurol Sci 1999;168:96-106. 